\title{
SPATIAL STRUCTURE OF THE GLOBULAR CLUSTER SYSTEM AROUND NGC 4472
}

\author{
EUNHYEUK KIM, MYUNG GYOON LEE \\ Department of Astronomy, Seoul National University \\ Seoul, 151-742, Korea; ekim@astro.snu.ac.kr
}

AND

DOUG GEISLER

KPNO/NOAO,USA

NGC 4472 (M49) is a giant elliptical galaxy in the Virgo cluster, and has a rich GCS. Radial surface density profiles of the GCS in this galaxy are well-known(Cohen 1988, Harris 1986). However, little information is available for the ellipticity $(e)$ and position angles of the GCS in NGC 4472. In this study we have investigated in detail the spatial structures of the GCS in NGC 4472 (the surface density, the ellipticity, the color, and the position angle) in comparison with those of the halo, using a large sample of the globular clusters located in a wide field $\left(16^{\prime} \times 16^{\prime}\right)$ of NGC 4472 .

In the color-magnitude diagram of the point sources in the NGC 4472 image (see in Lee, Kim \& Geisler, 1995), the objects with the colors of $1.0<$ $\left(C-T_{1}\right)<2.3$ are considered to be globular clusters in NGC 4472 . To avoid the possible contamination in the faint end due to the faint background galaxies we have selected only the GCs brighter than $T_{1}=22.5$ mag for the following analyses. The total number of GCs in our sample is $\sim 1,300$. The sample of the GCs has been divided into two groups ( the metal-poor GC $([\mathrm{Fe} / \mathrm{H}]<-0.5)$ and the metal-rich $\mathrm{GC}([\mathrm{Fe} / \mathrm{H}]>-0.5))$ to investigate the differences between the two groups.

Radial distribution of the surface density of the GC shows that the metal-rich GC is spatially more concentrated than the metal-poor GC ( the core radii of the metal-rich GC and the metal-poor GC are measured to be $\sim 140^{\prime \prime}$ and $\sim 240^{\prime \prime}$, respectively). The ellipticity of the metal-rich clusters decreases from $e \sim 0.8$ to $e \sim 0$ within $R=150^{\prime \prime}$ as the galactocentric 


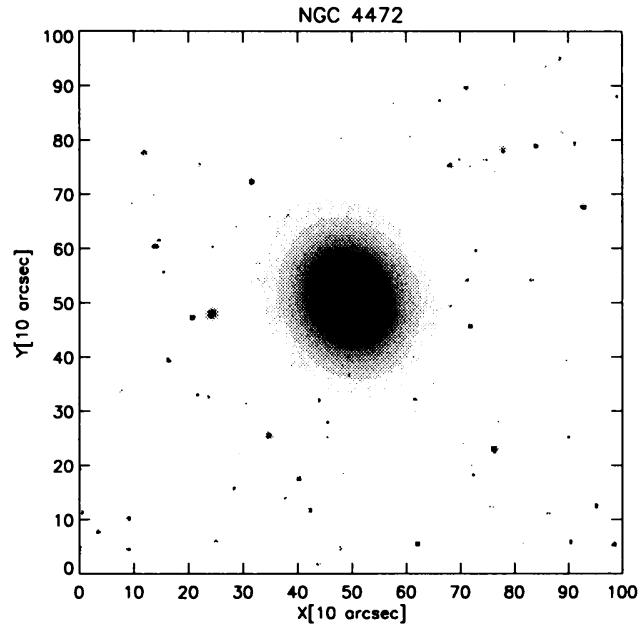

Figure 1. A grayscale map of $T_{1}$ $\left(T_{\text {exp }}=60 \mathrm{sec}\right)$ image of NGC 4472 . North is at top, and east is to the right.

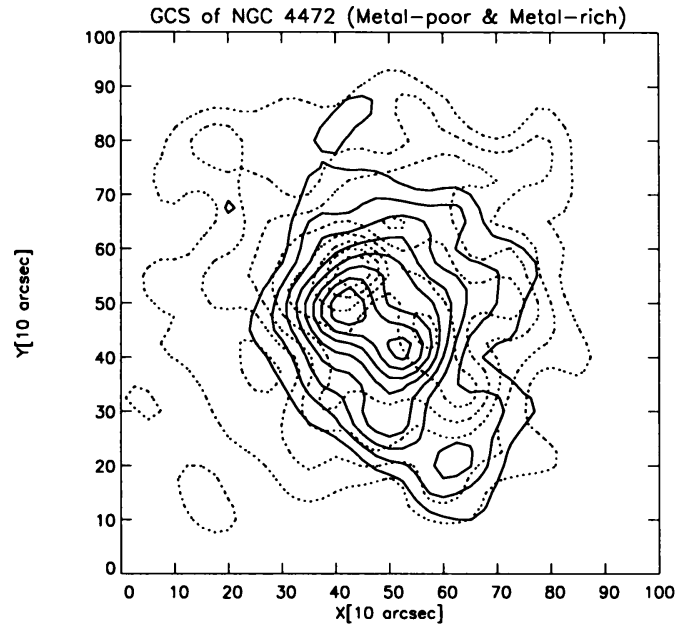

Figure 2. Displayed is the surface density contour map of the metal-poor GCs (dotted line) and the metal-rich GCs(solid line). Note that the distribution of the metal-rich GCs is spatially more concentrated than that of the metal-poor GCs.

radius increases, and increases to $e \sim 0.4$ outward beyond $R=150^{\prime \prime}$, while the ellipticity of the metal-poor clusters stays approximately constant at $e \sim 0.4$. The position angles (PA's) of the two groups show a significant difference in the inner region $\left(R<300^{\prime \prime}\right)$, while the PA's of the two groups agree approximately $\left(\mathrm{PA} \sim 140^{\circ}\right)$ in the outer region $\left(R>300^{\prime \prime}\right)$. These differences suggest that they have different formation histories.

The galaxy halo light shows quite different features from the GCs. The surface brightness of the galaxy halo light decreases more steeply than that of the GC. The median colors of the GCs get bluer outward. On the contrary the colors of the halo get bluer by a small amount outward in the inner region $\left(R<180^{\prime \prime}\right)$ and become much redder outward beyond $R=180^{\prime \prime}$. The mean color of the halo is much redder than that of the GC. The ellipticity of the halo remains almost constant with a value of $e \sim 0.2$, and the PA of the halo decreases slightly outward $\left(\mathrm{PA} \sim 160^{\circ}\right)$. These results imply that the halo and the GCs in NGC 4472 formed and evolved via different processes.

\section{References}

Cohen, J. G., 1988, AJ, 95, 682

Harris, W. E., 1986, AJ, 91, 822

Lee, M. G., Kim, E., \& Geisler, D., 1995, this proceeding, in press 